\title{
A disseminação de conteúdos em periódicos: propriedades bibliométricas, representações e medidas
}

\author{
Guido Rummler \\ Professor pleno. Universidade Estadual de Feira de Santana (UEFS) \\ - BA. Pós-doutorado pela USP. \\ E-mail: guido.rummler@gmail.com
}

\begin{abstract}
Resumo
Compreende-se por disseminação bibliométrica um fenômeno que está relacionado à situação de veiculação de um conteúdo em diferentes unidades bibliográficas. O presente estudo objetiva estabelecer um indicador a ser utilizado na análise da disseminação de, por exemplo, citações a autores ou a publicações, de metodologias ou técnicas, de terminologias, editais, assuntos, etc. O estudo tem por subjacência o que é enunciado como "lei da distribuição de conteúdos em periódicos". A disseminação é graficamente representada segundo níveis de distribuição (revista, volume, fascículo, artigo) do objeto de análise. São identificadas quatro propriedades da disseminação (espalhamento, expansividade, cronicidade e penetrabilidade), matematicamente representadas por coeficientes. Estes compõem um "índice de disseminação ponderada (IDP)", assim adjetivado por atender diferenças hierárquicas quanto à natureza dos elementos bibliográficos que contemplam o objeto de análise. Em que pese a

multidimensionalidade conjugada nesse indicador (4 variáveis abrangendo os domínios físico, temporal e cognitivo) sua utilização compreende baixa complexidade, demonstrada por aplicação a um exemplo empírico.
\end{abstract}

Palavras-chave

Disseminação. Indicadores. Bibliometria. Periódicos. Cientometria. Informetria.

\section{Contents' dissemination in journals: bibliometric properties, representations and measures}

\begin{abstract}
Bibliometric dissemination is regarded as a phenomena related to the distribution pattern of a content in different bibliographic elements. The present study aims to establish an indicator applicable in the dissemination analysis of publication or author citations, dissemination of certain methodology or technique, terms, themes, edicts, etc. As underlying the study a "law of content's distribution in periodicals" is enunciated. The dissemination is graphically represented in terms of distribution levels [periodical, volume (year), issue (number), article] of the object of analysis. Four properties of dissemination are identified (spreading, chronically, expansively and penetration), mathematically expressed by coefficients. These coefficients compose an "index of ponderal dissemination (IPD)" regarding hierarchical differences between the bibliographic elements. Although the multidimensionality (4 variables embracing physical, temporal and cognitive domains) the application of IPD represents low complexity according evidenced by an empirical example.
\end{abstract}

\section{Keywords}

Dissemination. Indicators. Bibliometrics. Journals. Scientometrics. Informetrics.

\section{INTRODUCุÃO}

Disseminação, difusão e dispersão são termos comumente utilizados para designar um processo, ou sua conseqüência, que é o fato de algo estar distribuído por diferentes partes. São termos utilizados em ciências da informação também indistintamente. No presente estudo, será entendido por disseminação bibliométrica um fenômeno, factível de ser avaliado, relacionado à situação de distribuição de um conteúdo segundo diferentes unidades bibliográficas de suporte. Em se tratando de formas de analisar este fenômeno no âmbito da bibliometria, encontra-se, nas bases dessa, o modelo matemático de Bradford sobre a concentração/dispersão de assuntos na literatura científica, geralmente denominado "Lei de Bradford", abordada em relação a distintas facetas segundo Ravichandra Rao (1996), Matos (2002), Ruiz e Jorge (2002) ou Guedes e Borschiver (2006), entre outros. Já Godin (1998) còmpôs um índice de dispersão com o propósito de medir o grau de difusão ou visibilidade de pesquisadores fora de seu próprio país.

Por outro lado, considerando que estudos, como, por exemplo, da dispersão geográfica de periódicos, são feitos por análise, geralmente dois a dois, de indicadores unidimensionais, tem-se a indicação de Wormell (1998), citada por Frandsen (2005), de que tais análises deveriam ser baseadas em diversos parâmetros. Porém, segundo Bufrem e Prates (2005), um modo de inovar os procedimentos cientométricos tem sido a utilização de indicadores multidimensionais. Tais autores, citando Sanz e Martín (1997), na página 16, caracterizam esses indicadores em função de "ter em conta, de forma simultânea, as distintas variáveis ou múltiplas interações observáveis nos documentos". Considerando essas questões, o presente estudo trata do desenvolvimento de um algoritmo para análise da disseminação de conteúdos em periódicos, relacionando quatro variáveis que, conjugadas, representam o fenômeno contemplando os domínios físico, temporal e da cognição, segundo identificação conceitual dos elementos quantificados.

\footnotetext{
${ }^{1}$ Ampliação de estudo prévio apresentado no $8^{\circ}$ Congresso Regional de Ciências da Saúde (CRICS), Rio de Janeiro, 16-19 de setembro de 2008.
} 
Na presente descrição, a designação "unidade de análise" (UA) será empregada segundo Singlenton et alii (1988), citados por Frota (1998), como objetos ou eventos aos quais as pesquisas sociais se referem, o que ou quem será descrito, analisado ou comparado. Em outros termos, segundo McGrath (1996), UA é o objeto de estudo. Compreende-se, portanto, que UA bibliométricas podem ser autores, textos, revistas, compêndios, terminologias, técnicas, metodologias, editais, citações etc. na condição de objetos de avaliação.

Pode-se dizer que uma relação entre variáveis ou mesmo uma representação unidimensional do modo de ser ou estar de uma UA constitui o que denominamos indicador. Desse modo, o presente estudo trata de buscar uma representação da disseminação de UAs como acima identificadas, com base em parâmetros quantificáveis a partir das referências bibliográficas dos textos que contemplam a referida unidade. Assim, é pretendido atender a uma representação multidimensional, conseqüentemente abrangente, da disseminação de conteúdos em publicações periódicas.

\section{METODOLOGIA}

O presente estudo compreende duas partes. A primeira relaciona-se à construção de indicadores quantitativos do fenômeno da disseminação, com base nas três fases identificadas por Trzesniak (1998):

a) busca, no processo, de dimensões ou aspectos com características específicas que possam, direta ou indiretamente, conter as respostas desejadas: padronização da metodologia de obtenção;

b) fase de reelaboração, por exemplo, reordenando os dados de diversos modos ou efetuando algum tipo de cálculo para que a informação desejada apareça; interpretação: o que a informação resultante significa;

c) fase do aperfeiçoamento da relação indicadorinformação: refinamento da etapa anterior; identificação de valores específicos dotados de significado especialmente relevante.

A segunda parte refere-se à aplicação empírica e análise das propriedades dos indicadores utilizando um exemplo sobre a disseminação dos assuntos/descritores "dengue" e "febre amarela" em revistas científicas nacionais. Os procedimentos específicos dessa aplicação são descritos no exemplo.

\section{Significados e relações entre elementos bibliográficos}

A ABNT (Associação Brasileira de Normas Técnicas, 2002) define uma referência como "um conjunto padronizado de elementos descritivos retirados de um documento que permite sua identificação individual". Em geral, os elementos utilizados para tal identificação são os mesmos nos diversos sistemas de referência, como, por exemplo, das normas ISO, Vancouver, ABNT etc., embora apresentem variações quanto à forma ou ordenamento em sua redação. No caso da referência relativa a publicações periódicas, seu título, número do correspondente volume (ou ano da revista), número do fascículo (ou número da revista) e as páginas ocupadas pelo texto referenciado constituem elementos localizadores da respectiva matéria. Assim, entende-se que por esses elementos se possa estabelecer o grau de disseminação bibliográfica de conteúdos publicados nestes suportes. Em relação à natureza desses elementos de identificação/localização bibliográfica, pode-se assim defini-los:

- periódico, um ente nominal caracterizado por sua natureza informacional, cuja concretude é dada pelos fascículos;

- artigo (ou similar), uma unidade informacional ímpar, espacialmente delimitada em um fascículo de revista;

- fascículo, uma parcialidade física e cronologicamente ímpar de uma revista, que caracteriza, pelo seu conteúdo, a natureza da mesma;

- volume de revista, um marcador que delimita abrangência etária (geralmente anual) das emissões periódicas de uma revista;

Tem-se assim, em suma, que, enquanto os volumes de uma revista referem temporalidade (cronologia) e a revista, domínio cognitivo (áreas de conhecimento), o artigo compreende os domínios cognitivo (temática) e físico (espaço), sendo que no fascículo a esses se acrescenta o temporal (periodicidade). Conjugações dois a dois desses elementos (figura 1) definem as seguintes propriedades da disseminação:

- do espalhamento, expressa por relação entre a quantidade "a" de artigos e a quantidade "f" de fascículos em que se encontra distribuída a unidade de análise de um estudo;

- da cronicidade, resultante da relação entre o número "v" de volumes e respectiva quantidade de fascículos que veiculam a unidade de análise; 
- da expansividade, expressa por uma relação entre a quantidade " $\mathrm{r}$ " de revistas e o número de volumes em que se encontra a unidade de análise;

- da penetrabilidade, estabelecida por relação entre a quantidade de artigos e de revistas que os publicam.

Outrossim, destaque-se que as propriedades identificadas, exceto cronicidade, contemplam mais de um domínio dimensional, segundo os parâmetros envolvidos e antes caracterizados.

\section{Representação gráfica e o estabelecimento de indicadores}

Uma representação gráfica da disseminação bibliométrica de uma unidade de análise (UA) qualquer é dada na figura 1 pela plotagem ortogonal das variáveis a (número de artigos), f (número de fascículos), v (número de volumes) e r (quantidade de revistas), conforme contagem de suas ocorrências na correspondente lista das referências bibliográficas dos textos que contemplam a UA. O quadrante em que se estabelece a relação de a com $\mathrm{f}$ corresponde a espalhamento; o de f com v, a cronicidade; o de v com $\mathrm{r}$ a expansividade e o de $\mathrm{r}$ com a a penetrabilidade.

Os vetores $\mathrm{E}_{\mathrm{s}} \mathrm{C}_{\mathrm{r},} \mathrm{E}_{\mathrm{x}}$ e $\mathrm{P}_{\mathrm{e}}$ podem ser, respectivamente, representados pelos pares ordenados $(A, F),(F, V),(V, R)$ e $(\mathrm{R}, \mathrm{A})$.

\section{FIGURA 1}

Relações entre parâmetros da distribuição de uma unidade de análise localizada em "a" artigos, "f” fascículos e "v" volumes de " $r$ " revistas, e respectivas conotações bibliométricas.

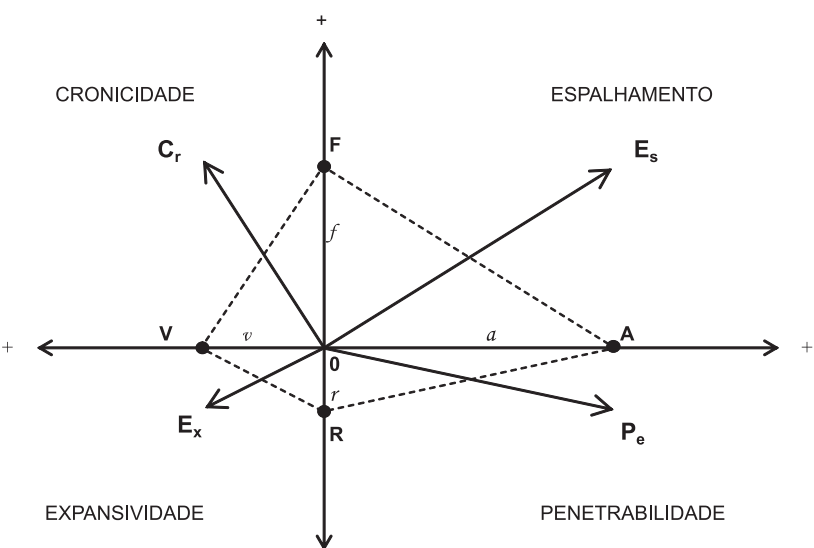

Por outro lado, cada vetor (distância de cada ponto desde a origem dos eixos) é igual à hipotenusa do respectivo triângulo retângulo cujos catetos são as coordenadas do correspondente vetor. O conjunto dos triângulos constitui um quadrilátero, por diante designado "polígono de disseminação".

Qualquer variação na medida de algum dos parâmetros (catetos) altera a configuração de dois triângulos contíguos e, conseqüentemente, do polígono de disseminação.

Considerando que triângulos diversos podem apresentar a mesma medida de área ou a mesma medida de algum lado, por razões a serem posteriormente evidenciadas, esses serão metricamente representados pela relação área/ hipotenusa. As expressões dessa relação respectivas a cada quadrante são:

Referente ao espalhamento:

$$
\frac{a \cdot f}{2 \sqrt{a^{2}+f^{2}}}
$$

Referente à cronicidade:

$$
\frac{f \cdot v}{2 \sqrt{f^{2}+v^{2}}}
$$

Refrente à expansividade:

$$
\frac{\mathrm{v} \cdot \mathrm{r}}{2 \sqrt{\mathrm{v}^{2}+\mathrm{r}^{2}}}
$$

Referente à penetrabilidade:

$$
\frac{r \cdot a}{2 \sqrt{r^{2}+a^{2}}}
$$

Entretanto, se cada uma dessas expressões for multiplicada (fator de normalização) por $2 \sqrt{2}$, ter-se-á como resultado um coeficiente cuja medida corresponderá a 1 quando a UA for encontrada em apenas um artigo, isto é, quando $\mathrm{a}=\mathrm{f}=\mathrm{v}=\mathrm{r}=1$, ou será igual ao valor de um parâmetro quando este for igual ao seu par na respectiva expressão. Portanto,

Coeficiente normalizado de espalhamento, $\mathrm{CE}_{\mathrm{S}}=\frac{\mathrm{a} \cdot \mathrm{f} \sqrt{2}}{\sqrt{\mathrm{a}^{2}+\mathrm{f}^{2}}}$

Coeficiente normalizado de cronicidade,

$\mathrm{CC}_{\mathrm{r}}=\frac{\mathrm{f} \cdot \mathrm{v} \sqrt{2}}{\sqrt{\mathrm{f}^{2}+\mathrm{v}^{2}}}$

Coeficiente normalizado de expansividade,

$\mathrm{CE}_{\mathrm{x}}=\frac{\mathrm{v} \cdot \mathrm{r} \sqrt{2}}{\sqrt{\mathrm{v}^{2}+\mathrm{r}^{2}}}$

Coeficiente normalizado de penetrabilidade,

$\mathrm{CP}_{\mathrm{e}}=\frac{\mathrm{r} \cdot \mathrm{a} \sqrt{2}}{\sqrt{\mathrm{r}^{2}+\mathrm{a}^{2}}}$ 
Conforme adiante será demonstrado, tais expressões conferem aos respectivos coeficientes caráter de ponderabilidade segundo o grau de hierarquia existente entre os pares de elementos.

Considerando ainda que o polígono de disseminação (figura 1) é constituído pelos quatro triângulos, poderse-á caracterizá-lo pela soma dos respectivos coeficientes, denominando a média destes coeficientes índice de disseminação ponderada (IDP). Portanto,

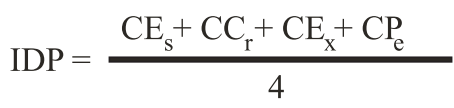

Aspectos comportamentais dos indicadores e a "lei da distribuição de conteúdos em periódicos”

Sendo "r" a quantidade de revistas das quais há uma quantidade "v" de volumes representados por uma quantidade "f" de fascículos que tem "a" artigos que contemplam uma unidade de análise, ter-se-á:

a) que sua distribuição obedecerá ao que por ser irrefutável, pode ser chamado de "lei da distribuição de conteúdos em periódicos”. Corresponde à seguinte relação:

$$
r \leqslant v \leqslant f \leqslant a
$$

Em outros termos, essa lei diz que inexiste a possibilidade de serem encontrados mais fascículos do que a quantidade de artigos neles contidos veiculando a UA. Da mesma forma não podem haver mais volumes que fascículos ou mais revistas que volumes contemplando a UA. Portanto, na representação gráfica do polígono de disseminação (figura1), também inexiste a possibilidade de troca de medidas entre catetos de tamanho diverso;

b) que, por decorrência da lei citada, os coeficientes ajustados apresentam as seguintes margens de variação:

$$
\begin{aligned}
& 1 \leqslant \mathrm{CE}_{\mathrm{s}} \leqslant \mathrm{a} \\
& 1 \leqslant \mathrm{CC}_{\mathrm{r}} \leqslant \mathrm{f} \\
& 1 \leqslant \mathrm{CE}_{\mathrm{x}} \leqslant \mathrm{V} \\
& 1 \leqslant \mathrm{CP}_{\mathrm{e}} \leqslant 1 \mathrm{a}
\end{aligned}
$$

c) que a seqüência dos elementos artigo-fascículovolume-revista constitui uma escala de elementos com hierarquização ascendente ( $<$ F $<$ V $<$ ) na representação da disseminação, enquanto as quantidades referentes a esses elementos obedecem à distribuição escalar da lei acima citada.

E que, por isso, variações de igual quantidade que ocorram, por vez, em cada parâmetro da medida de um fator de disseminação, promoverão efeito diverso. Portanto, produzirá maior repercussão a variação aplicada ao elemento de maior hierarquia, que, exceto em situação de igualdade com seu par, é o que apresenta menor valor. Isso ocorre em função da relação citada referente a duas propriedades matemáticas. Uma do produto e outra da potenciação.

Quanto à primeira, sendo $\mathrm{a}>\mathrm{f}$ e $\mathrm{n}_{1}$ um valor de acréscimo:

$$
\left(a+n_{1}\right) f<a\left(f+n_{1}\right)
$$

Quanto à segunda, sob igual condição:

$$
\left(a+n_{1}\right)^{2}+f^{2}>a^{2}+\left(f+n_{1}\right)^{2}
$$

Desse modo, na expressão, por exemplo, do coeficiente de espalhamento

$$
\mathrm{CE}_{\mathrm{s}}=\frac{\mathrm{a} \cdot \mathrm{f} \sqrt{2}}{\sqrt{\mathrm{a}^{2}+\mathrm{f}^{2}}},
$$

o efeito do aumento de f, no dividendo dessa expressão, é maior que igual aumento em "a”. O contrário acontece no divisor. Portanto, $\mathrm{CE}_{\mathrm{S}}$ sofrerá maior impacto por aumento no elemento hierárquico maior $\mathrm{f}$ (ao qual corresponde menor contagem, quando a \# $\mathrm{f}$, segundo a lei da distribuição de conteúdos em periódicos"). Tais ocorrências estarão evidenciadas no exemplo empírico adiante apresentado;

d) que cada parâmetro tem participação dupla na variação da disseminação. A ponderabilidade de um termo está em função da posição hierárquica que apresenta em relação a seu par na respectiva propriedade da disseminação. Por exemplo, o fascículo tem ascendência sobre o artigo como componente do fator de espalhamento, enquanto que, como componente do fator de cronicidade tem sobre si a ascendência do volume. Por outro lado, a revista é o elemento de ascendência na constituição de dois fatores: da expansividade e da penetrabilidade. Já o número de artigos tem menor peso nos dois fatores de que participa (espalhamento e penetrabilidade). Considere-se outrossim que, por razão dessas diferenças hierarquizantes entre os pares de elementos que compõem o índice geral, é apropriado adjetivá-lo como ponderado (índice de disseminação ponderada); 
e) conforme poderá ser verificado no exemplo empírico, a ponderabilidade intrínseca ao índice de disseminação (IDP) é demonstrada pelo fato de que sua medida é menor que a média aritmética dos elementos que o constituem (exceto quando $\mathrm{a}=\mathrm{f}=\mathrm{v}=\mathrm{r}$ ), porquanto, em cada parcela do indicador, o elemento de menor valor (nível hierárquico maior) tem maior peso que seu par. Assim,

$$
r \leqslant \text { IDP } \leqslant \frac{a+f+v+r}{4}
$$

E, portanto,

$$
1 \leq \mathrm{IDP} \leq \mathrm{a}
$$

\section{EXEMPLO DE APLICAC̣̃̃O}

Para ilustrar peculiaridades do IDP, considerou-se adequada sua aplicação a assuntos de ocorrência variada na literatura. Sob tal expectativa, foram escolhidos dengue e febre amarela, visto que, como eventos de saúde pública de ocorrência episódica têm, por outro lado, o mesmo agente transmissor.

\section{Procedimentos e resultados}

A partir de um levantamento bibliográfico realizado por consulta à Biblioteca Virtual em Saúde (BVS) (< $\underline{\text { http: } / /}$

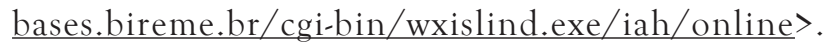
Acesso em: 9 set. 2008), cobrindo o período de 1986 a 2005, foram identificados 247 artigos contemplando assunto/descritor "dengue" apontados pelas bases BDENF, LILACS, ADOLEC e REPIDISCA.

Tendo por assunto/descritor "febre amarela", foram identificados 78 artigos dentre os registrados nas bases REPIDISCA, ADOLEC e LILACS.

Foram então constituídas listas das referências bibliográficas respectivas a cada assunto, segundo quadriênios de publicação, e feita a contagem tanto do número de artigos (a) quanto dos diferentes fascículos (f), diferentes volumes (v) e diferentes revistas (r) envolvidos em cada quadriênio.

Aplicando-se os escores obtidos por quadriênio às expressões para cálculo dos coeficientes de disseminação e índice de disseminação ponderada, foram obtidos os valores constantes nas tabelas 1 e 2 .

\section{TABELA 1}

Quantidade de suportes que contemplam o descritor/assunto "dengue” e respectivos indicadores de sua disseminação em cinco quadriênios.

\begin{tabular}{c||c|c|c|c||c|c|c|c|c}
\hline \multicolumn{1}{c||}{} & \multicolumn{4}{c||}{ SUPORTES } & \multicolumn{5}{c}{ INDICADORES * } \\
\cline { 2 - 15 } & $\mathbf{a}$ & $\mathbf{f}$ & $\mathbf{v}$ & $\mathbf{r}$ & $\mathrm{CE}_{\mathrm{s}}$ & $\mathrm{CC}_{\mathrm{r}}$ & $\mathrm{CE}_{\mathrm{x}}$ & $\mathrm{CP}_{\mathrm{e}}$ & IDP $^{2}$ \\
\hline $1986-1989$ & 13 & 10 & 10 & 10 & 11,21 & 10,00 & 10,00 & 11,21 & 10,60 \\
\hline $1990-1993$ & 30 & 29 & 23 & 16 & 29,48 & 25,49 & 18,57 & 19,96 & 23,38 \\
\hline $1994-1997$ & 40 & 37 & 27 & 13 & 38,41 & 30,85 & 16,56 & 17,48 & 25,83 \\
\hline $1998-2001$ & 94 & 61 & 47 & 20 & 72,36 & 52,65 & 26,02 & 27,67 & 44,67 \\
\hline $2002-2005$ & 70 & 59 & 43 & 25 & 63,82 & 49,14 & 30,56 & 33,30 & 44,20 \\
\hline
\end{tabular}

* $\mathrm{CEs}=$ coeficiente de espalhamento; $\mathrm{CCr}=$ coeficiente de cronicidade; $\mathrm{CEx}=$ coeficiente de expansividade; CPe = coeficiente de penetrabilidade; IDP = índice de disseminação ponderada. 
TABELA 2

Quantidade de suportes que contemplam o descritor/assunto "febre amarela" e respectivos indicadores de sua disseminação em cinco quadriênios.

\begin{tabular}{|c|c|c|c|c|c|c|c|c|c|}
\hline \multirow{2}{*}{ QUADRIÊNIO } & \multicolumn{4}{|c|}{ SUPORTES } & \multicolumn{5}{|c|}{ INDICADORES * } \\
\hline & a & $\mathrm{f}$ & $\mathbf{v}$ & $\mathbf{r}$ & $\mathrm{CE}_{\mathrm{s}}$ & $\mathrm{CC}_{\mathrm{r}}$ & $\mathrm{CE}_{\mathrm{x}}$ & $\mathrm{CP}_{\mathrm{e}}$ & IDP \\
\hline $1986-1989$ & 5 & 5 & 5 & 5 & 5,00 & 5,00 & 5,00 & 5,00 & 5,00 \\
\hline $1990-1993$ & 15 & 13 & 11 & 10 & 13,89 & 11,87 & 10,46 & 11,77 & 12,00 \\
\hline $1994-1997$ & 15 & 13 & 12 & 10 & 13,89 & 12,46 & 10,86 & 11,77 & 12,24 \\
\hline $1998-2001$ & 22 & 21 & 19 & 13 & 21,48 & 19,92 & 15,17 & 15,83 & 18,10 \\
\hline $2002-2005$ & 21 & 21 & 16 & 10 & 21,00 & 18,00 & 11,99 & 12,77 & 15,94 \\
\hline
\end{tabular}

* $\mathrm{CEs}=$ coeficiente de espalhamento; $\mathrm{CCr}=$ coeficiente de cronicidade; $\mathrm{CEx}=$ coeficiente de expansividade; CPe = coeficiente de penetrabilidade; IDP = índice de disseminação ponderada.

\section{Considerações}

Comparando-se os valores de IDP das duas unidades de análise, verifica-se igual tendência quanto à sua evolução temporal, embora seja constante a supremacia do índice referente à dengue.

Dentre os aspectos que foram levantados teoricamente quanto ao comportamento dos indicadores enunciados, tem-se aqui corroboração empírica de que:

- em uma distribuição homogênea IDP = a. Isto é, IDP $=5$, porque $\mathrm{a}=\mathrm{f}=\mathrm{v}=\mathrm{r}=5$ (tabela 2, 1º quadriênio);

- o valor correspondente a cada coeficiente é menor que a média entre seus dois elementos constituintes. Isso demonstra que a expressão de cada coeficiente confere maior peso ao elemento de maior hierarquia (menor escore), exceto quando houver igualdade entre os pares. Conseqüentemente, a média entre os quatro coeficientes, isto é, o IDP será menor que a média de a, f, v, r, cabendo-lhe, portanto, ser adjetivado como indicador ponderado;

- assim, variação em parâmetro de maior hierarquia tem efeito maior que igual variação em seu par no valor do respectivo coeficiente de disseminação. Logo, conforme a tabela 1 (quadriênios 2 e 3) e figura 2, o aumento de 23 para 27 no número de volumes não inverte o efeito da diminuição de 16 para 13 do número de revistas, causando uma diminuição de 18,57 para 16,56 no valor do $\mathrm{CE}_{\mathrm{X}}$.

De modo análogo, uma diminuição (tabela 1, quadriênios 4 e 5) de 24 artigos é contraposta por um aumento de apenas cinco revistas, assim ocorrendo aumento de 27,67 para 33,30 no valor do $\mathrm{CP}_{\mathrm{e}}$.
FIGURA 2

Polígonos de disseminação bibliométrica do assunto/descritor dengue em dois períodos.

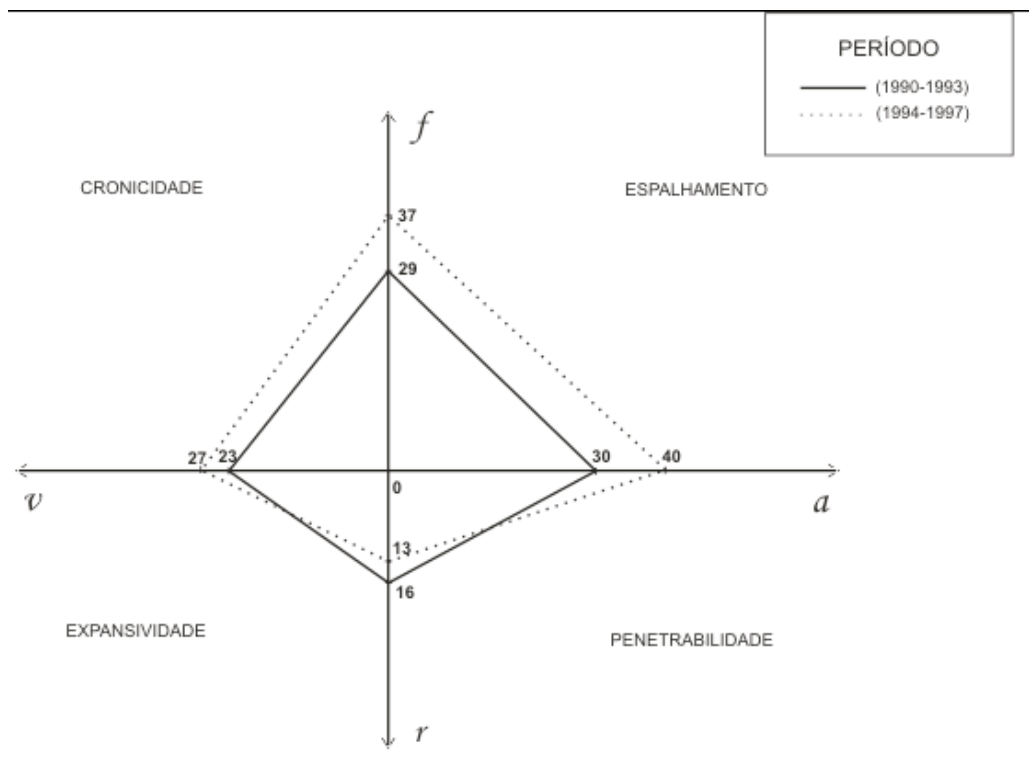




\section{DISCUSSÃO}

De acordo com Stubbs (2004), as normas ISO 11620 de 1998 definem genericamente indicadores como uma expressão numérica, simbólica ou verbal usada para caracterizar atividades (eventos, objetos ou pessoas) tanto em termos quantitativos quanto qualitativos para avaliar o valor das atividades caracterizadas e o método associado. Entretanto, Baena, Cases e Medina (2005) alertam sobre o cuidado a se ter quanto ao adjetivar indicadores como qualitativos ou quantitativos, por haver diferença de critérios entre autores. Embora ele considere como mais acertado seguir o critério do método de obtenção da informação, aponta três outras possibilidades: o critério do processo estatístico utilizado para análise da informação; o critério do objeto da medida e o critério da natureza das variáveis estatísticas utilizadas. Assim, de acordo com o primeiro critério, há de se considerar o IDP como um indicador quantitativo do fenômeno disseminação a ser qualitativamente interpretado (domínios abrangidos).

Considerando de modo amplo, Sem (2005) caracteriza um indicador bibliométrico como um dispositivo (estratégia) baseado em algum mecanismo de informação (usualmente informação bibliográfica), sendo uma ferramenta conceitual para facilitar projeções e a determinação do estado de uma atividade intelectual, constituindo, em última análise, um índice ou estatística.

Atendendo a preceitos anteriormente citados, o IDP peculiariza-se, ainda, segundo três aspectos:

1) considere-se que as medidas das variáveis podem, a critério do pesquisador, estar referidas a dados de uma coleção padrão de revistas, categorizando o indicador como relativo, segundo classificação de Vinkler (2001) e que tem o fator de impacto do ISI (Institut for Scientific Information) como um dos exemplos de maior repercussão, ou basear-se, como aqui, em busca aberta a periódicos que contemplam a UA. Tal aspecto, aliás, constitui-se em vantagem sobre o índice de dispersão segmentar (IDS) (RUMMLER, 2006) antes desenvolvido. É que na aplicação daquele, conforme demonstrada em análise da dispersão do impacto de revistas (RUMMLER, 2007), os escores referentes a a, $\mathrm{f}, \mathrm{v}$ e $\mathrm{r}$ são relativizados ao cômputo total desses elementos na coleção de referência. Entretanto, uma análise comparativa sobre a dispersão longitudinal (evolução temporal) ou vertical (entre duas UA, por exemplo), mediante aplicação do IDP, embora laboralmente facilitada em relação ao índice anterior, deve ser, assim como naquele, especificada sua correspondência à extensão temporal e bibliográfica elegida.

2) Considerando que uma UA contemplada, por exemplo, por quatro artigos, publicados em dois fascículos de duas revistas, não se encontra igualmente disseminada quanto se estivesse em quatro fascículos de uma mesma revista, estabeleceu-se a questão: como representar quantitativamente as diferenças hierárquicas entre revistas, volumes, fascículos e artigos em relação ao que representam como suportes de disseminação?

Essa questão tem certa analogia com recente crítica de Frandsen (2004) feita sobre a proposição de um fator de difusão de revistas (Journal Diffusion Factor - JDF) elaborado por Rowlands (2002), em que, a título de corrigir o excessivo peso representado pelo número de citações a uma revista, considerava que devesse ser levado em conta, em vez deste, o número de revistas citantes.

Essa questão-chave (ponderabilidade) trazida ao presente estudo levou à busca de determinações matemáticas baseadas na representação geométrica da distribuição das UAs, conforme descrita no item 2.2 e caracterizada no item 2.3 , que contempla uma representação ponderal da disseminação, aspecto a ser considerado como de aproximação de uma representação com a realidade representada. Tal realidade, porém, está adstrita à expressão $a \geq f \geq v \geq r$, que aqui foi enunciada como "lei da distribuição de um conteúdo em periódicos”. Esse enunciado foi assim classificado por atender ao conceito de lei expresso por Portal e Quesada (1997, p.90): "Aquilo que se define como a identificação de uma relação causal, constante e objetiva na natureza, sociedade ou pensamento".

Acrescente-se que uma lei deva ter caráter de indubitabilidade, o que aqui se identifica no fato de, por exemplo, três artigos diferentes poderem estar distribuídos por dois fascículos, mas que o número de fascículos não pode ser maior que a quantidade de artigos neles publicados.

3) Embora o presente estudo não utilize as técnicas (mapeamento) identificadas por Sanz e Martin (1997) como pertinentes a indicadores multidimensionais, o indicador aqui obtido é assim adjetivado dada sua natureza: um conjugado de quatro variáveis abrangendo domínios físico, temporal e cognitivo. Isso, porém, atende ao como aqueles autores caracterizam indicadores multidimensionais: "permitem ter em conta de forma simultânea as distintas variáveis ou as múltiplas inter- 
relações que podem ser observadas nos documentos, ou nos hábitos e necessidades de informação dos usuários" (p. 61).

Ressalte-se que o algoritmo apresentado reduz a uma só medida a representação da disseminação contemplando a hierarquização dos níveis de distribuição em que se encontra a UA.

\section{CONCLUSÕES}

Tendo por substrato o que este estudo enuncia como "lei da distribuição de conteúdos em periódicos" assim representada: $\mathrm{a} \geq \mathrm{f} \geq \mathrm{v} \geq \mathrm{r}$, em que a é a quantidade de artigos, f de fascículos, $v$ de volumes e $r$ de revistas que contemplam um conteúdo ou UA, foram deduzidas as seguintes expressões que representam propriedades da disseminação:

- coeficiente de espalhamento, $\quad \mathrm{CE}_{\mathrm{s}}=\frac{\mathrm{a} \cdot \mathrm{f} \sqrt{2}}{\sqrt{\mathrm{a}^{2}+\mathrm{f}^{2}}}$

- coeficiente de cronicidade, $\quad \mathrm{CC}_{\mathrm{r}}=\frac{\mathrm{f} \cdot \mathrm{v} \sqrt{2}}{\sqrt{\mathrm{f}^{2}+\mathrm{v}^{2}}}$

- coeficiente de expansividade, $\quad \mathrm{CE}_{\mathrm{x}}=\frac{\mathrm{v} \cdot \mathrm{r} \sqrt{2}}{\sqrt{\mathrm{v}^{2}+\mathrm{r}^{2}}}$

- coeficiente de penetrabilidade, $\quad \mathrm{CP}_{\mathrm{e}}=\frac{\mathrm{r} \cdot \mathrm{a} \sqrt{2}}{\sqrt{\mathrm{r}^{2}+\mathrm{a}^{2}}}$

- a média desses coeficientes constitui o "índice de disseminação ponderada (IDP)

Logo, $\quad$ IDP $=\frac{\mathrm{CE}_{\mathrm{s}}+\mathrm{CC}_{\mathrm{r}}+\mathrm{CE}_{\mathrm{x}}+\mathrm{CP}_{\mathrm{e}}}{4}$

- o menor valor possível para o IDP é 1. O valor máximo possível corresponde ao número de artigos "a";

- o IDP, exceto nas situações limites acima, apresenta valores menores que a média referente aos quatro parâmetros a, f, v e a, em razão de constituir representação que pondera diferenças hierárquicas entre os distintos níveis de distribuição da unidade de análise;

- o IDP constitui uma representação multidimensional, conjugando 4 variáveis que contemplam os domínios físico, temporal e cognitivo;
- sua aplicação é feita com base no corpus de uma pesquisa que é constituído pelas referências bibliográficas das unidades (textos artigos etc.) que contemplam os conteúdos (citações, autor, terminologias, técnicas, periódicos, assuntos, editais, etc.) cuja disseminação se quer avaliar;

- o algoritmo aqui apresentado envolve baixa complexidade operativa e acurácia na diferenciação de situações de distribuição do conteúdo avaliado.

Artigo submetido em 27/11/2008 e aceito em 26/12/2008.

\section{REFERÊNCIAS}

ASSOCIACT̃̃O BRASILEIRA DE NORMAS TÉCNICAS - ABNT. NBR 6023: informação e documentação: referências: elaboração. Rio de Janeiro, 2002.

BAENA, A. J. L.; CASES, M. V.; MEDINA, M. B. Indicadores cuantitativos y cualitativos para la evaluación de la actividad investigadora: ¿complementarios? ¿contradictorios? ¿excluyentes? Cuadernos IRC. Disponível em: <http://www2.uca.es/serv/ consejo social/doc/ transferencia14.pdf $>$ Acesso em: 28 jun. 2005.

BUFREM, L.; PRATES, Y. O saber científico registrado e as práticas de mensuração da informação. Ciência da Informação, Brasília, v. 34, n. 2, p. 9-25, 2005.

FRANDSEN, T. F. Journal diffusion factors: a measure of diffusion? Aslib Proceedings, v. 56, n. 1, p. 5-11, 2004.

FROTA, M.G.C. A delimitação das unidades de análise em ciência da informação. Ciência da Informação, Brasília, v. 27, n. 3, p. 262-267, set./dez. 1998.

GODIN, B. Measuring knowledge flows between countries: the use of scientific meeting data. Scientometrics, v. 42, n. 3, p. 313-323, 1998.

GUEDES, V. L. S.; BORCHIVER, S. Bibliometria: uma ferramenta estatística para a gestão da informação e do conhecimento, em sistemas de informação, de comunicação e avaliação científica e tecnológica. Disponivel em: <http://www.cinform.ufba.br/vi anais/docs/VaniaLS Guedes.pdf $>$. Acesso em: 24 maio 2006.

MATOS, N. E. P. La bibliografía, bibliometría y las ciencias afines. ACIMED, v. 10, n. 3, 2002. Disponível em: 〈http://www.bvs.sld.cu/ revistas/aci/vol10 3 02/Aci012002.htm> Acesso em: 07 jun. 2005.

McGRATH, W. E. The unit of analysis (objects of study) in bibliometrics and scientometrics. Scientometrics, v. 35, n. 2, p. 257-264, 1996.

PORTAL, S. G.; QUESADA, E. S. Las supuestas "leyes" métricas de la información. Revista General de Información y Documentación, v. 7, n. 2, p. $87-93$.

RAVICHANDRA RAO, I. K. An analysis of Bradford multipliers and a model to explain law of scattering. Scientometrics, v. 41 , n. 1/2, p. 98 $100,1998$. 
A disseminação de conteúdos em periódicos: propriedades bibliométricas, representações e medidas*

ROWLANDS, I. Journal diffusion factors: a new approach to measuring research influence. Aslib Proceedings, v. 54, n. 2, 2002.

RUIZ, J. A. A.; JORGE, R. A. Informetría, bibliometría y cienciometría : aspectos teórico-prácticos. ACIMED, v. 10, n. 4. Disponível em: $<$ http://eprints.rclis.org/archive/00001654 > Acesso em: 09 mar. 2007.

RUMMLER, G. Modelagem de um indicador bibliométrico para análise da dispersão de conhecimentos. Ciência da Informação, Brasília, v. 35, n. 1, p. 63-71, 2006

Comparando periódicos pela medida da dispersão de seu impacto. Perspectivas em Ciência da Informação, v. 12, n. 2, p. 118-129, 2007.

SANZ CASADO, E.; MARTIN MORENO, C. Técnicas bibliométricas aplicadas a los estudios de usuarios. Revista General de Información y Documentación, v. 7, n. 2, p. 41-68, 1997.
SEM, S. K. For what purpose are the bibliometric indicators and how should they work. Disponivel em: 〈http://www.ricyt.org/interior/ normalizacion/IV taller/sen.pdf $>$ Acesso em: 18 jul. 2005.

STUBBS, E. A. Indicadores de desempeño: naturaleza, utilidad y construcción. Ciência da Informação, Brasília, v. 33, n. 1, p. 149-154, 2004.

TRZESNIAK, P. Indicadores quantitativos: reflexões que antecedem seu estabelecimento. Ciência da Informação, Brasília, v. 27, n. 2, p. 159-164, 1998.

VINKLER, P. An attempt for defining some basic categories of scientometrics and classifying the indicators of evaluative scientometrics. Scientometrics, v. 50, n. 3, p. 539-544, 2001.

WORMELL, I. Geographical concentration: the case of economics journals. Scientometrics, v. 63, n. 1, p. 69-95, 2005. 